\title{
A systematic review of non-motor symptom evaluation in clinical trials for amyotrophic lateral sclerosis
}

\author{
Emily Beswick ${ }^{1,2,3}$ (1) $\cdot$ Deborah Forbes ${ }^{1,2,3} \cdot$ Zack Hassan $^{1,2,3} \cdot$ Charis Wong $^{1,2,3}$ (D) Judith Newton ${ }^{1,2,3} \cdot$ Alan Carson $^{1}$. \\ Sharon Abrahams ${ }^{3,4}$. Siddharthan Chandran ${ }^{1,2,3,4,5}$. Suvankar Pal ${ }^{1,2,3}$
}

Received: 4 March 2021 / Revised: 28 May 2021 / Accepted: 4 June 2021 / Published online: 13 June 2021

(c) The Author(s) 2021

\begin{abstract}
Background Amyotrophic lateral sclerosis (ALS) is increasingly recognised as a multi-system disorder, presenting with common and impactful non-motor symptoms, such as neuropsychiatric symtpoms, cognitive and behavioural changes, pain, disordered sleep, fatigue and problematic saliva.

Aim/hypothesis We aimed to systematically review 25 years of ALS clinical trials data to identify if non-motor features were evaluated, in addition to the traditional measures of motor functioning and survival, and where evaluated to describe the instruments used to assess. We hypothesised that assessment of non-motor symptoms has been largely neglected in trial design and not evaluated with ALS-suitable instruments.

Methods We reviewed clinical trials of investigative medicinal products in ALS, since the licensing of riluzole in 1994. Trial registry databases including WHO International Trials Registry, European Clinical Trials Register, clinicaltrials.gov, and PubMed were systematically searched for Phase II, III or IV trials registered, completed or published between 01/01/1994 and 16/09/2020. No language restrictions were applied.

Results 237 clinical trials, including over 29,222 participants, were investigated for their use of non-motor outcome measures. These trials evaluated neuropsychiatric symptoms $(75,32 \%)$, cognitive impairment $(16,6.8 \%)$, behavioural change (34, $14 \%)$, pain $(55,23 \%)$, sleep disturbances $(12,5 \%)$ and fatigue $(18,8 \%)$. Problematic saliva was assessed as part of composite ALS-FRS(R) scores in 184 trials (78\%) but with no focus on this as an isolated symptom. 31 (13\%) trials including 3585 participants did not include any assessment of non-motor symptoms.

Conclusions Non-motor symptoms such as neuropsychiatric, cognitive and behavioural changes, pain, disordered sleep, fatigue, and problematic saliva have not been consistently evaluated in trials for people with ALS. Where evaluated, nonsymptoms were primarily assessed using instruments and impairment thresholds that are not adapted for people with ALS. Future trials should include non-motor symptom assessments to evaluate the additional potential therapeutic benefit of candidate drugs.
\end{abstract}

PROPSERO registration CRD42020223648.

Keywords Amyotrophic lateral sclerosis $\cdot$ Clinical trials $\cdot$ Non-motor symptoms $\cdot$ Outcome measures

Suvankar Pal

Suvankar.Pal@ed.ac.uk

1 Centre for Clinical Brain Sciences, The University of Edinburgh, Edinburgh, Scotland, UK

2 Anne Rowling Regenerative Neurology Clinic, The University of Edinburgh, 49 Little France Crescent, Edinburgh EH16 4 SB, Scotland, UK
3 Euan MacDonald Centre for MND Research, The University of Edinburgh, Edinburgh, Scotland, UK

4 Human Cognitive Neurosciences, Psychology, School of Philosophy, Psychology and Language Sciences, The University of Edinburgh, Edinburgh, Scotland, UK

5 UK Dementia Research Institute, The University of Edinburgh, Edinburgh, Scotland, UK 


\section{Introduction}

The focus of assessment and symptom management in amyotrophic lateral sclerosis (ALS) is traditionally on limb weakness, speech and swallowing difficulties, and respiratory failure. Despite this, a range of other symptoms are repeatedly reported as impactful and poorly evaluated in people with ALS (pwALS) including neuropsychiatric symptoms, cognitive and behavioural changes, pain, disordered sleep, fatigue, and problematic saliva [1].

These symptoms are often collectively termed 'nonmotor' or 'extra-motor' $[1,2]$ and result in significant functional impairment, reduced quality of life (QoL), higher disease burden, and negative prognoses [2-6]. People with ALS experiencing greater frequency of nonmotor symptoms report lower quality of life than those who indicate more severe motor symptoms, suggesting that the impact of these non-motor symptoms on the daily lives of people with ALS is comparable to, if not greater, than of motor symptoms [2,3]. These symptoms can arise secondary to motor dysfunction, such as inefficient saliva clearance from bulbar motor dysfunction, and pain from inability to regularly move and turn. Symptoms may localise elsewhere neuroanatomically [2] broadening our understanding of the aetiopathogenesis of ALS and providing insights into wider neuroanatomical dysfunction [7]. Clinical management [8] and trial design guidelines for ALS [9] have evolved to incorporate evaluation and treatment of non-motor symptoms as part of holistic assessment of ALS [10].

Our previous work has reported how neuropsychiatric, cognitive and behavioural assessments have been employed as outcome measures and exclusion criteria in ALS trials [11]. This identified that these aspects were under-evaluated in trial design, and often using measures unsuitable, or not adapted for, people with progressive disability. In this study, we intend to broaden our scope to include other non-motor symptoms important in ALS: pain, sleep disturbance, fatigue, and problematic saliva. In addition, we will evaluate the assessment tools used as outcome measures in greater detail, we have continued to include neuropsychiatric, cognitive and behaviour assessment in this review to provide a complete picture of nonmotor evaluation.

\section{Non-motor symptoms in clinical care and trial design}

The focus on motor symptoms in clinical and research contexts is likely impacted by the limited availability of disease-modifying drugs for people with ALS. Riluzole is currently the only globally licensed disease-modifying therapy for ALS with limited efficacy, resulting in prolongation of survival by $2-3$ months [12]. There is a significant unmet need in therapeutic options for people affected by ALS.

To deliver holistic disease management for people with ALS, it is necessary to expand our conceptualisation of 'treatment' beyond improved physical function and extended survival. Effective management, or ultimately slowed progression, of non-motor symptoms due to pharmacological intervention should be evaluated as part of any novel investigative medicinal products' efficacy in clinical trials [13]. Inclusion of alternative outcome measures to evaluate potential impact of candidate drugs on non-motor impairment is recommended as a potential area of consideration for trial design in the current Airlie House guidelines, which focus on ALS-specific trial development [9]. The potential beneficial effect of candidate drugs which successfully manage non-motor features of a debilitating condition may have significant clinical impact, improving quality of life, reducing disability and disease burden.

The method of assessment is also of particular relevance in trials of people with ALS. Due to progressive disability, overlap with somatic symptoms, disease-specific impairments and speech decline, traditional measures may not be as effective in detecting change in symptoms, directly reducing their suitability to evaluate people with ALS [1]. This can be mediated through using tools which are validated specifically for this cohort $[14,15]$, or tools with revised impairment thresholds [16] which account for the specific profile of impairment characterised by ALS. In this systematic review of non-motor outcome measures in ALS trials, we will consider the types of assessment tools used and their suitability to evaluate non-motor presentations in this population.

\section{Aims and hypotheses}

We aimed to systematically review historical clinical trials of interventional medicinal products (IMPs) in ALS, since the licensing of riluzole in 1994, to identify if non-motor features of ALS were evaluated as outcome measures. In addition, we aimed to review the assessment tools used, their characteristics and suitability for evaluating nonmotor symptoms in people with ALS. We hypothesised that non-motor symptoms have been largely overlooked in trial design and that where evaluated, assessed with instruments that are not specifically designed to evaluate symptoms in this population. 


\section{Methods}

We completed a systematic, unbiased, search of trial registries including clinicaltrials.gov, World Health Organisation's (WHO) International Clinical Trials Registry Platform (ICTRP), European Union Clinical Trials Register (EduraCT) and PubMed on 16/09/2020 for Clinical Trials of an Investigational Medicinal Product (CTIMP). Using the search terms "amyotrophic lateral sclerosis" or "motor neuron* disease", we searched clinicaltrials.gov for interventional trials of investigative medicinal products. We searched European Union Clinical Trials Register (EudraCT) and WHO International Clinical Trials Registry Platform (ICTRP) for trials of "amyotrophic lateral sclerosis" with the filters "Phase II", "Phase III" and "Phase IV" applied. Using the advanced search feature, we filtered PubMed with ("amyotrophic lateral sclerosis"[MeSH Terms] OR "motor neuron* disease" [MeSH Terms]). We then applied the 'Clinical Trial' filter for Article Type, Human trials only and Publication Date within the criteria defined above.

Phase II, III or IV trials assessing potential diseasemodifying therapies in subjects with amyotrophic lateral sclerosis that were registered, completed or published between 01/01/1994 and 16/09/2020 were included. No language restrictions were applied. Extension trials, post hoc analysis papers, stem cell therapies, imaging studies, medical device studies, non-ALS subjects and trials focussed on symptom management were excluded.

\section{Data extraction}

The following details of selected trials were extracted "Investigational Medicinal Product (IMP) Assessed", "Number of Participants", "Date of Commencement", "Primary Outcome Measure(s)" and "Secondary Outcome Measure(s)". We reviewed each assessment tool used as an exclusion criteria or outcome measure in the included trials to explore whether they evaluated non-motor symptoms; defined in this study as neuropsychiatric, cognitive impairment, behavioural changes, pain, disordered sleep, fatigue, and problematic saliva. Each assessment tool was categorised as ALS specific, symptom specific, both ALS and symptom specific or generic in content focus. We then reviewed each trial included in this review for their use of each assessment tool and subsequent evaluation of each non-motor symptom.

Each assessment tool was reviewed and data extracted on the intended focus of assessment, administrator (clinician or self-report), if the scoring is affected by the presence of motor disability or speech impairment and the time to administer. We also explored the availability of diseasespecific impairment thresholds where applicable, and the availability of non-English translations.

\section{Results}

\section{Overview}

The search identified 1507 records, (PRISMA diagram in Fig. 1). 353 were removed due to duplication and 907 did not meet inclusion criteria (defined in Fig. 1); in particular results which were not clinical trials of investigative medicinal products and non-ALS subjects. 237 clinical trials remained. These trials were proposed to include over 29,222 trial participants with ALS. The non-motor symptoms evaluated in this review are neuropsychiatric, cognitive impairment, behavioural changes, pain, disordered sleep, fatigue, and problematic saliva. Table 1 summarises the reported prevalence of these symptoms in the ALS population, and the pharmacological and non-pharmacological treatments suggested with evidence derived from a Cochrane database systematic review of treatments [10] and United Kingdom National Institute for Clinical Excellence (NICE) clinical care guidelines [8]. The trials forming the main dataset of this review are focussed on therapeutic targets for motor symptoms and survival improvement. Table 2 provides a summary of how frequently each non-motor symptom considered in this review was evaluated in the clinical trials. These seven non-motor symptoms were included as outcome measures or evaluated within quality of life measures (QoL) in 206 trials (87\%). Neuropsychiatric symptoms were assessed in 75 trials (32\%) and cognitive impairment was evaluated as an outcome measure in 16 trials (6.8\%) Behavioural change was evaluated in 33 trials (14\%), pain in 55 trials (23\%) and fatigue in 18 trials (8\%). Sleep disturbances were evaluated in 12 trials (5\%). Whilst saliva assessment was included in 184 trials (78\%), this was part of a composite measure embedded within the either the ALS-FRS-(R) (Amyotrophic Lateral Sclerosis Functional Rating Scale Revised [17]) or the CNS-BFS (Centre for Neurologic Studies Bulbar Function Scale [18]), and the impact of drugs of saliva problems was not assessed specifically. 31 trials (13\%) did not include any assessment of saliva, neuropsychiatric, cognitive impairment, behavioural changes, pain, disordered sleep and fatigue as an outcome measure or evaluate within a quality of life measure.

\section{Symptom evaluation}

\section{Neuropsychiatric symptoms}

Neuropsychiatric symptoms were assessed within quality of life measures in 61 trials (26\%); 29 (48\%) of these trials used 


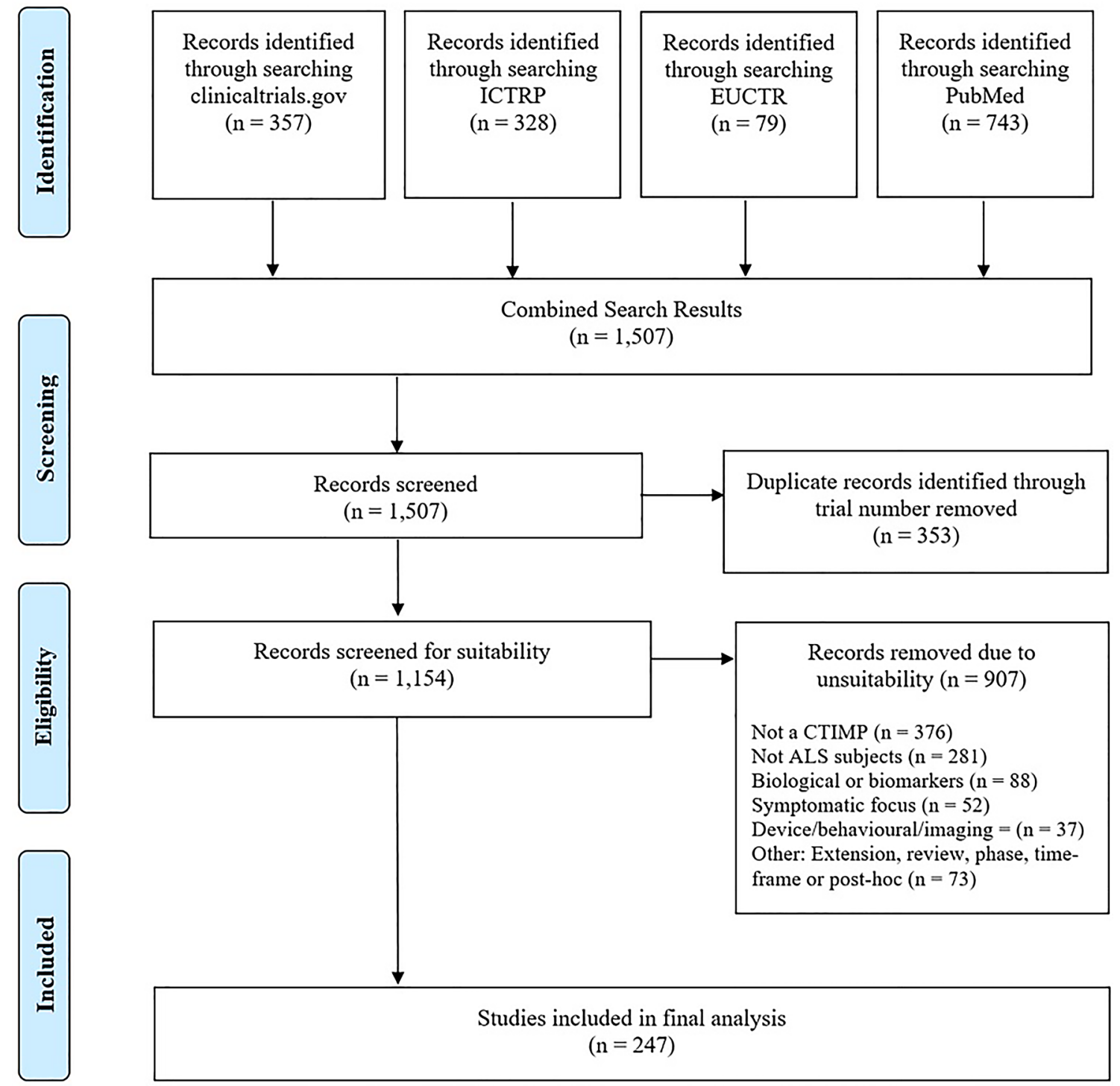

Fig. 1 PRISMA diagram for record selection process

ALS-specific quality of life measures, 29 (48\%) generic and 3 a combination. Four trials used a combination of generic quality of life measures and neuropsychiatric assessments that were not ALS specific. No data on assessment tool used were available for one trial.

Seven trials used neuropsychiatric measures which were not developed specifically for people with ALS; ESAS (Edmonton Symptom Assessment Scale [19]), HamiltonDepression [20], NPI-Q (Neuropsychiatric Inventory Questionnaire [21]) and C-SSRS (Columbia Suicide Severity Rating Scale [22]). Only one trial utilised an ALS-specific neuropsychiatric assessment of depression, the ADI-12 (ALS Depression Inventory [14]), in combination with the more widely used HADS (Hospital Anxiety and Depression Scale [23]), the unmodified version.
Trials evaluating neuropsychiatric symptoms within quality of life measures utilised: Edmonton Symptom Scale (ESS [19]), ALS-Specific Quality of Life (ALSSQOL-R [24]), ALS Assessment Questionnaire (ALSAQ [25]), Short Form Health Survey (SF [26]), EuroQol [27], McGill[28] and Sickness Impact Profile (SIP [29]). These quality of life measures did not provide separate scores for the neuropsychiatric symptoms evaluated. Items focussing on neuropsychiatric symptoms were often limited to binary assessment (present or absent) with scoring reported within the overall quality of life score, making change over time difficult to ascertain (Tables 3, 4). 


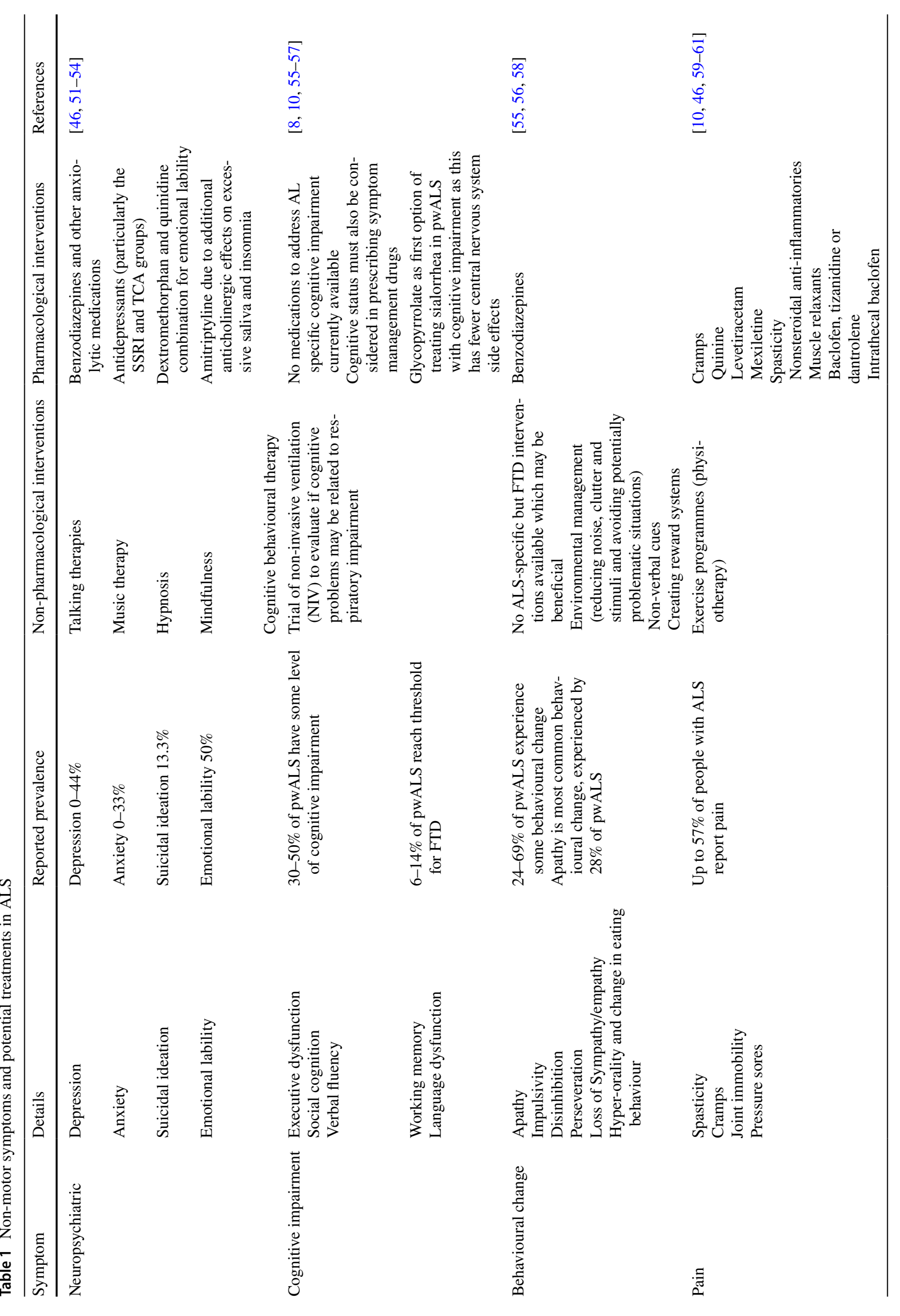




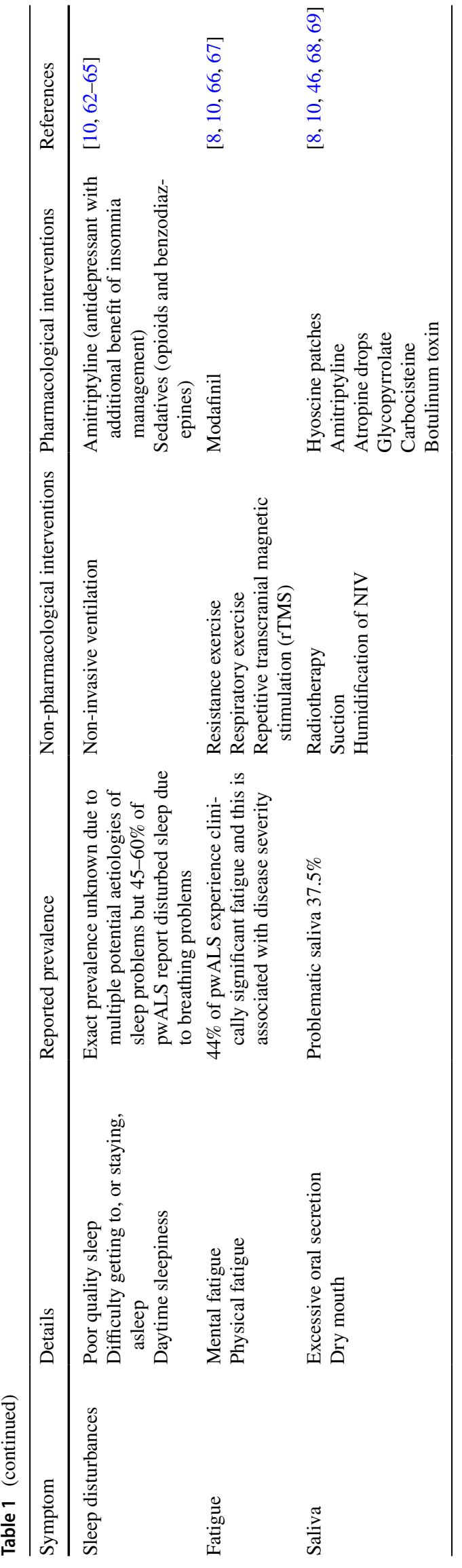

\section{Cognition and behaviour change}

Cognition was evaluated in 16 trials (7\%); within quality of life measures in 5 trials (Sickness Impact Profile/ALS19 and ALSSQOL-R). Seven trials used the ECAS (Edinburgh Cognitive Assessment Screen [15]) and one used the ALS-CBS (ALS Cognitive Behavioural Screen [30]), both ALS-specific measures of cognitive impairment. One of the seven trials using the ECAS also evaluated cognition using the MoCA (Montreal Cognitive Assessment [31]), a measure of global cognition that is not specifically designed for people with ALS. Two trials used the ACE-III (Addenbrooke's Cognitive Assessment [32]) and another a test of verbal fluency, both tests of cognitive function which are not disease specific.

People with ALS may lack insight into cognitive and behavioural changes [33], or downplay experiences due to stigma [34]. Objective measures (such as the ECAS, ALS-CBS, MoCA, ACE-III, and verbal fluency) focus on clinical evaluation and task-based assessments, whereas self-report measures (such as the SIP/ALS-19 and ALSSQOL-R) are reliant on the person with ALS to recognise and disclose their cognitive difficulties.

34 trials (14\%) evaluated behavioural symptoms in participants. Five of these were within the context of quality of life measures: SIP and ALS-19. Nine were within assessment tools also evaluating cognition, eight trials using measures such as the ECAS and ALS-CBS which are specifically designed for ALS and one trial using the FBI (Frontal Behavioural Inventory [35]), a non-diseasespecific assessment including behavioural items. Emotional lability is a key behavioural change experienced by some individuals with ALS and was evaluated in 19 trials; 18 of which used the Norris scale (which includes one item assessing emotional lability [36]) and one the visual analogue scale on emotionality.

\section{Pain}

Pain was evaluated in 55 trials (23\%). Assessment was included in the context of quality of life measures (such as the ALS Assessment Questionnaire, EuroQoL measures and ALS Quality of Life tools) in 46 trials. Frequency of reporting changes in levels of pain are not reported separately when evaluated in quality of life measures.

Cramp was specifically addressed in trials using questionnaires or visual analogue scales [37]. Other outcome measures evaluating pain utilised numeric rating scales, functional assessments (Edmonton Symptom Assessment Scale [19]) and quantification of pain-related adverse events (NCT03690791). 
Table 2 Assessments utilised and non-motor symptoms evaluated

\begin{tabular}{|c|c|c|c|c|c|c|c|}
\hline Tool & $\begin{array}{l}\text { Intended area of } \\
\text { focus }\end{array}$ & $\begin{array}{l}\text { Non-motor symp- } \\
\text { tom assessed }\end{array}$ & $\begin{array}{l}\text { Separate score } \\
\text { for non-motor } \\
\text { symptom? }\end{array}$ & ALS specific & $\begin{array}{l}\text { Symp- } \\
\text { tom } \\
\text { specific }\end{array}$ & $\begin{array}{l}\text { ALS and } \\
\text { symptom } \\
\text { specific }\end{array}$ & $\begin{array}{l}\text { Frequency of use } \\
\text { as an outcome } \\
\text { measure }^{\mathrm{a}}\end{array}$ \\
\hline $\begin{array}{l}\text { C-SSRS (Colum- } \\
\text { bia Suicide } \\
\text { Severity Rating } \\
\text { Scale) }\end{array}$ & Suicidality & Neuropsychiatric & Yes & No & Yes & No & 4 \\
\hline $\begin{array}{l}\text { NPI-Q (Neuropsy- } \\
\text { chiatric Inventory } \\
\text { Questionnaire) }\end{array}$ & Neuropsychiatric & Neuropsychiatric & Yes & No & Yes & No & 1 \\
\hline \multirow[t]{3}{*}{$\begin{array}{l}\text { ESS (Edmon- } \\
\text { ton Symptom } \\
\text { Assessment } \\
\text { Scale) }\end{array}$} & Quality of life & Neuropsychiatric & No & No & No & No & 1 \\
\hline & & Pain & No & No & No & No & 1 \\
\hline & & Sleep & No & No & No & No & 1 \\
\hline $\begin{array}{l}\text { HADS (Hospital } \\
\text { Anxiety and } \\
\text { Depression } \\
\text { Scale) }\end{array}$ & $\begin{array}{r}\text { Anxiety and } \\
\text { depression }\end{array}$ & Neuropsychiatric & Yes & No & Yes & No & 2 \\
\hline $\begin{array}{l}\text { ADI-12 (Amyo- } \\
\text { trophic Lateral } \\
\text { Sclerosis Depres- } \\
\text { sion Inventory-12 } \\
\text { item) }\end{array}$ & Depression & Neuropsychiatric & Yes & No & No & Yes & 1 \\
\hline $\begin{array}{l}\text { HAM-D (Hamil- } \\
\text { ton-Depression) }\end{array}$ & Depression & Neuropsychiatric & Yes & No & Yes & No & 3 \\
\hline \multirow[t]{5}{*}{$\begin{array}{l}\text { ALSSQOL-R } \\
\text { (Amyotrophic } \\
\text { Lateral Sclerosis } \\
\text { Specific Quality } \\
\text { of Life-Revised } \\
\text { and Short Form) }\end{array}$} & Quality of life & Pain & No & Yes & No & No & 33 \\
\hline & & Fatigue & No & Yes & No & No & 33 \\
\hline & & Neuropsychiatric & No & Yes & No & No & 33 \\
\hline & & Sleep & No & Yes & No & No & 33 \\
\hline & & Cognition $^{\mathrm{b}}$ & No & Yes & No & No & 33 \\
\hline \multirow[t]{2}{*}{$\begin{array}{l}\text { SF-8, SF-12 and } \\
\text { SF-36 (Short } \\
\text { Form Health } \\
\text { Survey-8 item, } \\
12 \text { item or } 36 \\
\text { item) }\end{array}$} & Quality of life & Pain & No & No & No & No & 31 \\
\hline & & Neuropsychiatric & No & No & No & No & 31 \\
\hline $\begin{array}{l}\text { SEI-QoL (Sched- } \\
\text { ule for Individual } \\
\text { Quality of Life) }\end{array}$ & Quality of life & Self-reported (any) & No & No & No & No & 1 \\
\hline $\begin{array}{l}\text { ALSAQ-5 (Amyo- } \\
\text { trophic Lateral } \\
\text { Sclerosis Assess- } \\
\text { ment Question- } \\
\text { naire-5 item) }\end{array}$ & Quality of life & Neuropsychiatric & No & Yes & No & No & 6 \\
\hline $\begin{array}{l}\text { ALSAQ-40 } \\
\text { (Amyotrophic } \\
\text { Lateral Sclero- } \\
\text { sis Assessment } \\
\text { Question- } \\
\text { naire-40 item) }\end{array}$ & Quality of life & Pain & No & Yes & No & No & 34 \\
\hline
\end{tabular}


Table 2 (continued)

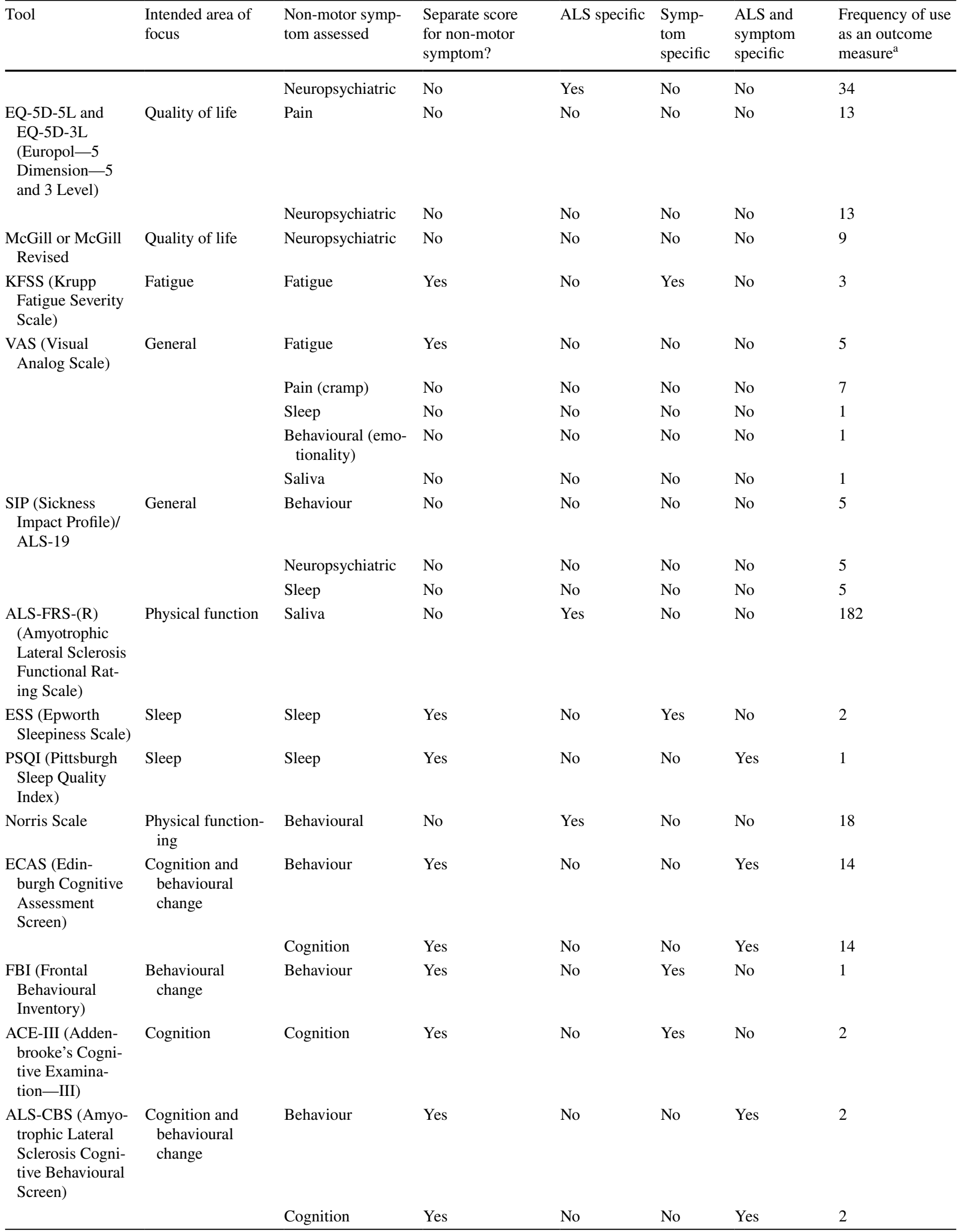


Table 2 (continued)

\begin{tabular}{|c|c|c|c|c|c|c|c|}
\hline Tool & $\begin{array}{l}\text { Intended area of } \\
\text { focus }\end{array}$ & $\begin{array}{l}\text { Non-motor symp- } \\
\text { tom assessed }\end{array}$ & $\begin{array}{l}\text { Separate score } \\
\text { for non-motor } \\
\text { symptom? }\end{array}$ & ALS specific & $\begin{array}{l}\text { Symp- } \\
\text { tom } \\
\text { specific }\end{array}$ & $\begin{array}{l}\text { ALS and } \\
\text { symptom } \\
\text { specific }\end{array}$ & $\begin{array}{l}\text { Frequency of use } \\
\text { as an outcome } \\
\text { measure }^{\text {a }}\end{array}$ \\
\hline $\begin{array}{l}\text { MoCA (Mon- } \\
\text { treal Cognitive } \\
\text { Assessment) }\end{array}$ & Cognition & Cognition & Yes & No & Yes & No & 1 \\
\hline Verbal Fluency & Cognition & Cognition & Yes & No & Yes & No & 1 \\
\hline $\begin{array}{l}\text { CNS-BFS (Centre } \\
\text { for Neurologic } \\
\text { Bulbar Function } \\
\text { Scale) }\end{array}$ & Physical function & Saliva & No & Yes & No & No & 2 \\
\hline $\begin{array}{l}\text { CNS-LS (Centre } \\
\text { for Neurologic- } \\
\text { Lability Scale) }\end{array}$ & Emotional lability & Behaviour & Yes & No & Yes & No & 1 \\
\hline $\begin{array}{l}\text { DSM (Diagnos- } \\
\text { tic Statistical } \\
\text { Manual) and } \\
\text { frontotemporal } \\
\text { dementia criteria } \\
\text { (FTD) }\end{array}$ & Diagnosis criteria & Behaviour & No & No & Yes & No & 2 \\
\hline \multirow{2}{*}{$\begin{array}{l}\text { Symptom-specific } \\
\text { Questionnaires }\end{array}$} & General & Pain (cramp) & Yes & No & Yes & No & 1 \\
\hline & & Fatigue & No & No & Yes & No & 1 \\
\hline $\begin{array}{l}\text { No Data on } \\
\text { Assessment Tool }\end{array}$ & $\begin{array}{l}\text { Emergent suicidal- } \\
\text { ity }\end{array}$ & Neuropsychiatric & No & No & No & No & 1 \\
\hline
\end{tabular}

aPlease be aware that this is the number of times used, not the number of trials, as some trials may have utilised several outcome measures to evaluate the same non-motor symptom or a single outcome measure several times

${ }^{\mathrm{b}}$ Indicates self-reported cognitive problems, not a formal clinician assessment of cognitive function

\section{Sleep}

13 trials (5.5\%) evaluated sleep, 9 of which utilised only quality of life measures. The quality of life measures did not provide a separate score for sleep-related symptoms as the scores were reported as an overall measure of quality of life. The remaining three trials used the symptomspecific Epworth Sleepiness Scale [38], visual analogue scales or the Pittsburgh Sleep Quality Index [39].

\section{Fatigue}

18 trials (7.6\%) evaluated fatigue as an outcome measure. Eight of these trials measured fatigue within quality of life measures and as a result, no separate scores for each nonmotor symptom were reported, only the aggregate score for quality of life. One trial utilised the ESAS, a generic tool to document change in patient-reported symptoms. Nine trials evaluated fatigue specifically, utilising visual analogue scales, presence/absence statements, the Krupp Fatigue Severity Scale [40].

\section{Problematic saliva}

182 trials $(77 \%)$ reported using the ALS-FRS or ALS-FRS(R), as an outcome measure that evaluates saliva within the context of physical function. Ten of these trials also utilised additional saliva evaluations: the CNS-BFS (Centre for Neurologic Studies Bulbar Function Scale), a visual analogue scale and the ALSSQOL-R (ALS Quality of Life Revised [41]). No disease and symptom-specific measures of saliva symptoms were included in the trials within this review. Neither the ALS-FRS nor the CNS-BFS provide scores for the severity or frequency of an individuals' saliva problems.

\section{Assessment tools}

Of the 237 trials included in this study which evaluated nonmotor symptoms, 49 versions or combinations assessment tools were used. In this study, we categorised assessment tools as ALS-specific (designed and validated specifically for people with ALS), symptom specific (focussing only on the non-motor symptom under consideration), both (disease and symptoms specific), and generic (evaluating the 
Table 3 Assessment tool suitability for ALS

\begin{tabular}{|c|c|c|c|c|c|c|c|}
\hline Domain & Tool acronym & Tool name & Administrator & $\begin{array}{l}\text { Scoring affected } \\
\text { by motor dis- } \\
\text { ability or speech } \\
\text { impairment? } \\
\text { (Yes/no) }\end{array}$ & $\begin{array}{l}\text { Disease-specific } \\
\text { impairment } \\
\text { threshold avail- } \\
\text { able? } \\
\text { (Yes/no) }\end{array}$ & $\begin{array}{l}\text { Available in } \\
\text { languages other } \\
\text { than English? } \\
\text { (Yes/no) }\end{array}$ & $\begin{array}{l}\text { Time to } \\
\text { administer } \\
\text { (min) }\end{array}$ \\
\hline Quality of life & ALSAQ-5/40 & $\begin{array}{l}\text { Amyotrophic } \\
\text { Lateral Sclero- } \\
\text { sis Assessment } \\
\text { Question- } \\
\text { naire-5 or } 40 \\
\text { item }\end{array}$ & $\begin{array}{r}\text { Clinician or } \\
\text { researcher }\end{array}$ & No & Yes & $\begin{array}{l}\text { Yes } \\
{[70]}\end{array}$ & $5-30$ \\
\hline Quality of life & SIP/ALS-19 & $\begin{array}{l}\text { Sickness Impact } \\
\text { Profile }\end{array}$ & $\begin{array}{l}\text { Self-report ques- } \\
\text { tionnaire }\end{array}$ & No & Yes & No & $10-20$ \\
\hline Quality of life & ALSSQOL & $\begin{array}{l}\text { Amyotrophic Lat- } \\
\text { eral Sclerosis } \\
\text { Specific Quality } \\
\text { of Life }\end{array}$ & $\begin{array}{l}\text { Self-report ques- } \\
\text { tionnaire }\end{array}$ & No & Yes & $\begin{array}{l}\text { Yes } \\
{[71]}\end{array}$ & $10-20$ \\
\hline Disease burden & ESAS & $\begin{array}{l}\text { Edmonton Symp- } \\
\text { tom Assessment } \\
\text { Scale }\end{array}$ & $\begin{array}{l}\text { Self-report ques- } \\
\text { tionnaire }\end{array}$ & No & No & $\begin{array}{l}\text { Yes } \\
{[72]}\end{array}$ & $5-10$ \\
\hline Quality of life & $\begin{array}{l}\text { EQ-5D-5L } \\
\text { EQ-5D-3L }\end{array}$ & EuroQol & $\begin{array}{l}\text { Self-report ques- } \\
\text { tionnaire }\end{array}$ & No & No & Yes & 5 \\
\hline Quality of life & McGill & McGill & $\begin{array}{r}\text { Clinician or } \\
\text { researcher }\end{array}$ & No & Yes & Yes & $10-20$ \\
\hline Physical & ALS-FRS-(R) & $\begin{array}{l}\text { Amyotrophic } \\
\text { Lateral Sclero- } \\
\text { sis Functional } \\
\text { Rating Scale }\end{array}$ & $\begin{array}{r}\text { Clinician or } \\
\text { researcher }\end{array}$ & No & Yes & Yes & $5-10$ \\
\hline Cognition & ACE-III & $\begin{array}{l}\text { Addenbrooke's } \\
\text { Cognitive } \\
\text { Examination } \\
\text { - III }\end{array}$ & $\begin{array}{r}\text { Clinician or } \\
\text { researcher }\end{array}$ & Yes & No & $\begin{array}{l}\text { Yes } \\
{[73]}\end{array}$ & $20-30$ \\
\hline $\begin{array}{l}\text { Cognition and } \\
\text { behaviour }\end{array}$ & ALS-CBS & $\begin{array}{l}\text { Amyotrophic } \\
\text { Lateral Sclero- } \\
\text { sis Cognitive } \\
\text { Behavioural } \\
\text { Scale }\end{array}$ & $\begin{array}{l}\text { Clinician or } \\
\text { researcher and } \\
\text { caregiver ques- } \\
\text { tionnaire }\end{array}$ & No & Yes & $\begin{array}{l}\text { Yes } \\
{[74]}\end{array}$ & $5-10$ \\
\hline $\begin{array}{l}\text { Cognition and } \\
\text { behaviour }\end{array}$ & ECAS & $\begin{array}{l}\text { Edinburgh } \\
\text { Cognitive ALS } \\
\text { Screen }\end{array}$ & $\begin{array}{l}\text { Clinician or } \\
\text { researcher and } \\
\text { caregiver semi } \\
\text { structured } \\
\text { interview }\end{array}$ & No & Yes & $\begin{array}{l}\text { Yes } \\
{[75,76]}\end{array}$ & $15-30$ \\
\hline Behaviour & FBI & $\begin{array}{l}\text { Frontal Behav- } \\
\text { ioural Inventory }\end{array}$ & $\begin{array}{l}\text { Caregiver ques- } \\
\text { tionnaire }\end{array}$ & No & No & $\begin{array}{l}\text { Yes } \\
{[77]}\end{array}$ & $5-10$ \\
\hline Behaviour & NS & $\begin{array}{l}\text { Norris Scale (bul- } \\
\text { bar sub-scale) }\end{array}$ & $\begin{array}{r}\text { Clinician or } \\
\text { researcher }\end{array}$ & No & No & $\begin{array}{l}\text { Yes } \\
{[78]}\end{array}$ & $10-20$ \\
\hline Cognition & VF & $\begin{array}{l}\text { Verbal fluency } \\
\text { (No data available } \\
\text { on type of test) }\end{array}$ & $\begin{array}{r}\text { Clinician or } \\
\text { researcher }\end{array}$ & Yes & Yes & Yes & $5-10$ \\
\hline Behaviour & CNS-BFS & $\begin{array}{l}\text { Centre for Neu- } \\
\text { rologic Studies } \\
\text { Behavioural } \\
\text { Scale }\end{array}$ & $\begin{array}{l}\text { Self-report ques- } \\
\text { tionnaire }\end{array}$ & No & No & No & $10-15$ \\
\hline Behaviour & CNS-LS & $\begin{array}{l}\text { Centre for Neu- } \\
\text { rologic Studies } \\
\text { Lability Scale }\end{array}$ & $\begin{array}{l}\text { Self-report ques- } \\
\text { tionnaire }\end{array}$ & No & No & No & 5 \\
\hline Sleep & PSQI & $\begin{array}{l}\text { Pittsburgh Sleep } \\
\text { Quality Index }\end{array}$ & $\begin{array}{l}\text { Self-report and } \\
\text { caregiver ques- } \\
\text { tionnaire }\end{array}$ & No & No & $\begin{array}{l}\text { Yes } \\
{[79]}\end{array}$ & $5-10$ \\
\hline
\end{tabular}


Table 3 (continued)

\begin{tabular}{|c|c|c|c|c|c|c|c|}
\hline Domain & Tool acronym & Tool name & Administrator & $\begin{array}{l}\text { Scoring affected } \\
\text { by motor dis- } \\
\text { ability or speech } \\
\text { impairment? } \\
\text { (Yes/no) }\end{array}$ & $\begin{array}{l}\text { Disease-specific } \\
\text { impairment } \\
\text { threshold avail- } \\
\text { able? } \\
\text { (Yes/no) }\end{array}$ & $\begin{array}{l}\text { Available in } \\
\text { languages other } \\
\text { than English? } \\
\text { (Yes/no) }\end{array}$ & $\begin{array}{l}\text { Time to } \\
\text { administer } \\
\text { (min) }\end{array}$ \\
\hline Sleep & ESS & $\begin{array}{l}\text { Epworth Sleepi- } \\
\text { ness Scale }\end{array}$ & $\begin{array}{l}\text { Self-report ques- } \\
\text { tionnaire }\end{array}$ & No & No & Yes & 5 \\
\hline Fatigue & KFS & $\begin{array}{l}\text { Krupp Fatigue } \\
\text { Severity Scale }\end{array}$ & $\begin{array}{l}\text { Self-report ques- } \\
\text { tionnaire }\end{array}$ & No & No & Yes & 5 \\
\hline
\end{tabular}

Table 4 Non-motor symptom evaluation summary

\begin{tabular}{|c|c|c|c|}
\hline Non-motor symptom & Assessment group & Assessment tools used to evaluate & $\begin{array}{l}\text { Frequency of use as an outcome } \\
\text { measure (\% of } 237 \text { total trials) }\end{array}$ \\
\hline \multirow[t]{2}{*}{ Neuropsychiatric } & QoL measures & $\begin{array}{l}\text { Sickness Impact Profile, SF-8/12/36, ALS-19, ALSSQOL, } \\
\text { ALSAQ-5/40, ESAS, EQ-5D-5L, EQ-ED-3L, McGill }\end{array}$ & \multirow[t]{2}{*}{$74(31)$} \\
\hline & Symptom-specific & ADI-12, HADS, Hamilton-Depression, Columbia Suicide & \\
\hline \multirow[t]{2}{*}{ Cognitive } & QoL measures & Sickness Impact Profile, ALS-19 & \multirow[t]{2}{*}{$16(6.8)$} \\
\hline & Cognitive & $\begin{array}{l}\text { ACE-III, ALS-CBS, ECAS, MoCA, DSM Criteria (used as demen- } \\
\text { tia diagnostic criteria), Verbal Fluency }\end{array}$ & \\
\hline \multirow[t]{2}{*}{ Behavioural } & $\begin{array}{l}\text { Quality of life } \\
\text { and caregiver } \\
\text { burden }\end{array}$ & $\begin{array}{l}\text { Sickness Impact Profile, ALS-19, Zarit Burden Interview, Car- } \\
\text { egiver Burden Inventory }\end{array}$ & \multirow[t]{2}{*}{$37(15)$} \\
\hline & Symptom-specific & $\begin{array}{l}\text { ALS-CBS, CNS-LS, ECAS, FTD Criteria, Emotionality Analogue } \\
\text { Scale, FBI, Norris Scale }\end{array}$ & \\
\hline \multirow[t]{2}{*}{ Pain } & Quality of life & ALSSQOL, ESAS, EQ-5D-5L/3L, SF-12/36, McGill, ALSAQ-40 & \multirow[t]{2}{*}{$55(23)$} \\
\hline & Pain & $\begin{array}{l}\text { Cramp Questionnaire, Numeric Rating Scale, Visual Analogue } \\
\text { Scale }\end{array}$ & \\
\hline \multirow[t]{2}{*}{ Sleep } & Quality of life & $\begin{array}{l}\text { ALSSQOL, Sickness Impact Profile, Edmonton Symptom Assess- } \\
\text { ment Scale }\end{array}$ & \multirow[t]{2}{*}{$12(5)$} \\
\hline & Sleep & $\begin{array}{l}\text { Visual Analogue Scale, Pittsburgh Sleep Quality Index, Epworth } \\
\text { Sleepiness Scale }\end{array}$ & \\
\hline \multirow[t]{2}{*}{ Fatigue } & Quality of life & ALSSQOL, Edmonton Symptom Assessment Scale & \multirow[t]{2}{*}{$187.6)$} \\
\hline & Fatigue & $\begin{array}{l}\text { Visual Analogue Scale, Presence vs Absence Questions, Krupp } \\
\text { Fatigue Severity Scale, Fatigue Severity Scale }\end{array}$ & \\
\hline Saliva & & ALS-FRS-(R), Visual Analogue Scale, ALSSQOL-R, CNS-BFS & $182(77)$ \\
\hline
\end{tabular}

symptom within a general measure, usually a quality of life questionnaire). Six instruments used were ALS-specific (designed and validated specifically for people with ALS), four were symptom specific, four were both disease and symptom specific, and seven were symptom-generic (evaluating the symptom within a general measure, e.g. QoL, and not specifically evaluating that symptom).

Versions of the ALS-FRS-(R) most frequently utilised (182 trials, 77\%). A complete list of the assessment tools used, and the frequency that they are included as outcome measures, is available in Table 2. 208 of the 237 trials (88\%) included in this study evaluated one of the listed non-motor symptoms. However, 102 (49\%) of these can be accounted for with the use of the ALS-FRS(R) as a primary or secondary outcome to evaluate physical progression, with a single item on hypersalivation.

\section{Quality of Life}

70 trials (30\%) included quality of life assessments as outcome measures. These quality of life measures frequently contained questions on non-motor symptoms such as mood, pain and fatigue. Often these questionnaires include only a single item evaluating the presence of this non-motor symptom, with no additional information on its impact on the individual, severity or change over time $[42,43]$.

In the trials included in this review, $20 \%$ of the times where non-motor symptoms were assessed, this occurred within a quality of life measure, rather than a scale 
specifically evaluating that symptom. As a result, often no score for the non-motor symptom is reported. Of the 70 trials that used a quality of life assessment as an outcome measure, only 21 included an additional tool to evaluate non-motor symptoms, which was not the ALS-FRS-(R).

A range of quality of life (QoL) measures were used as outcome measures in the trials included in this review. ALS-specific measures; Sickness Impact Profile ALS, ALS Assessment Questionnaires, ALS-Specific Quality of Life enable us to evaluate how the candidate drug affects aspects of the individual's life most likely to be affected by ALS. Disease- and symptom-specific measures are more likely to be sensitive and specific enough to detect changes, crucial in clinical trials.

However, findings from drug trials using disease-specific measures are limited in their comparability across neurological conditions. In comparison, more general assessments of quality of life and physical functioning such as the Edmonton Symptom Assessment Scale, Short Form Health Surveys, EuroQol measures, Schedule for Individual QoL and McGill enable researchers to compare findings with existing health-related quality of life and disability data, but at the potential cost of evaluating disease-specific impairment.

\section{Symptom-specific measures}

Neuropsychiatric outcome measure such as the NPI-Q are neither disease-specific nor symptom specific. Whilst useful to capture the potential presence of broad range of disorders, the utility of this measure to detect change over time is limited due to the dichotomous outcome of Yes/No to the presence of disorders. Symptom-specific measures such as the HADS, C-SSRS and HAM-D were used as outcome measures in other included trials, the suitability of these measures for people with ALS is uncertain, due to overlap with somatic symptoms and disease progression. This can be mediated through the use of revised disease-specific thresholds of impairment [16]. The ADI-12 is a brief measure of depressive symptoms, specifically designed and validated for people with ALS [14]; evidence base and comparability outside ALS is limited.

The ECAS and ALS-CBS are disease-specific measures of cognitive and behavioural symptoms in people with ALS. Designed and validated for use in this population, they focus on the aspects of cognition and behaviour which are most affected in this condition and are sensitive to detecting changes across repeated assessment [44]. The MoCA and ACE-III are measures of global cognition, whilst not specifically intended to evaluate cognitive impairment in people with ALS they may have utility as outcome measures in trials to detect potential changes. However, both of these assessments rely on drawing tasks to evaluate cognitive functioning, the scoring on which may be detrimentally affected by the physical progression characteristic of ALS.

Verbal fluency is a measure of a specific aspect of cognition, often affected in people with ALS, however, using this assessment in isolation may be insufficient to detect the broad range of cognitive function that can be affected by ALS progression.

As ALS exists on a disease spectrum with frontotemporal dementia (FTD) [45], measures of behaviours that are included in FTD diagnosis, such as the Frontal Behavioural Inventory and DSM criteria (Diagnostic and Statistical Manual), can be of relevance to evaluating behavioural symptoms in people with ALS, even those who do not meet diagnostic threshold for FTD. However, these measures may also miss the nuanced behaviours that can occur in the heterogeneous presentations of ALS. Emotional lability can be a commonly experienced symptom of bulbar dysfunction, measures such as the CNS-LS, are beneficial to evaluate disease- and symptom-specific outcomes.

Pain was primarily evaluated within quality of life measures, both ALS-specific and generic measures. Symptomspecific assessments of pain and cramp prevalence and severity were limited to visual analogue scales and Cramp Questionnaires, which may not be sufficiently objective to detect the nuanced changes occurring within the progression of ALS and the potential impact of a candidate drug.

Symptom-specific sleep measures used in the trials in this review are the Epworth Sleepiness Scale and Pittsburgh Sleep Quality Index are beneficial to understand participant perspective and acknowledge that sleep quality is greater than just time spent at rest. However, in ALS, disordered breathing and declining respiratory function can be a significant contributor to the multifactorial issue of sleep. In tools which are not ALS specific, the impact of respiratory symptoms may not be accounted for. In ALS, the Epworth Sleepiness Scale has the additional benefit of indicating the severity of respiratory symptoms.

Contribution of other symptoms and evaluation using symptom-specific scales is also of consideration when evaluating fatigue. Using symptom-specific outcome measures such as the Krupp Fatigue Severity Scale, and the FSS, may not reflect the interwoven contributions to the conceptualisation and causes of physical and mental fatigue experienced in ALS.

\section{Saliva assessments}

Whilst $184(78 \%)$ trials evaluated saliva, in these trials saliva was assessed in the context of a single sub-domain score of the ALS-FRS(R) or with other bulbar symptoms, alongside swallowing and speech, in the CNS-BFS. Impact of the candidate drug, separate saliva score and change in saliva problems were not reported. 
ALS-FRS-(R) includes a single item on hypersalivation. CNS-BFS also considers problematic saliva, and the Norris Scale an item on behavioural change. As a result, despite apparent frequent measurement of saliva, as the measurement is within the ALS-FRS-(R), we know little about the potential impact of these candidate drugs on the saliva symptoms which can have a significant affect upon people with ALS [6].

Of the 206 trials which included an assessment of any non-motor symptom, saliva (using larger physical function, quality of life or bulbar assessment tools) was the only non-motor symptom assessed in $102(49 \%)$ of these trials. Ultimately, excluding the use of the ALS-FRS(R) as a physical function outcome measure, including the single item on salivation, saliva problems were under-evaluated. Only 80 (44\%) of the 182 trials using the ALS-FRS-(R), evaluating saliva, assessed any additional non-motor symptom.

\section{Discussion}

\section{Overview}

As our conceptualisation of ALS broadens from a motoronly disorder to one of multi-system involvement, it is vital that clinical management guidelines and trial design continue to reflect this. Effective symptom management remains a major priority for ALS care, as stated in NICE 2016 care guidelines [8]. Clinical management and trial design guidance recommend the inclusion of symptom-focussed outcome measures to evaluate potential additional therapeutic benefits $[9,10]$.

This study considers the evaluation of non-motor symptoms as outcome measures in 237 trials proposed to include over 29,222 trial participants with ALS in the last 25 years, since the licensing of riluzole in 1994. The non-motor symptoms included in this review are neuropsychiatric symptoms, cognitive and behavioural changes, pain, disordered sleep, fatigue, and problematic saliva, all of which are prevalent in and impactful in ALS [1]. Our findings indicate that nonmotor symptoms were not consistently evaluated and where evaluated, assessment tools were not specific to ALS, or the non-motor symptom being evaluated.

\section{Evaluation and management}

Effective management and treatment of non-motor symptoms can have a significant impact on the lives of people with ALS and their caregivers, reducing disease burden and improving quality of life [8]. Non-motor symptoms can benefit from both non-pharmacological and pharmacological interventions and in conditions such as ALS where symptom management is currently the primary focus, managing these non-motor symptoms can benefit those living with ALS [46]. Using non-motor assessments in clinical evaluation of people with ALS can help us address these symptoms in care planning, disease management and when designing future research.

Under-evaluation of non-motor symptoms using disease specific measures is a potentially missed opportunity when considering the holistic impact of drug candidates on these troublesome symptoms. In our previous work, we found that neuropsychiatric and cognitive symptoms were consistently under-assessed in ALS trials [11]. Whilst improvement in motor functioning and prolonging survival remain the main goal in clinical drug trials, additional symptomatic benefit of candidate drugs can be of great interest. An additional benefit of including measures to evaluate these non-motor symptoms in trial design is a better understanding of the potential negative impact of candidate drugs on these aspects of ALS. An investigative medicinal product that may result in, or worsen existing, non-motor symptoms and in turn increase disease burden, may offset the potential improvement in motor symptoms for people with ALS. Greater knowledge of these side effects can help to inform licensing decisions and future suitability for prescription of the medication to sub-groups of the ALS population.

\section{Assessment tools}

In other neurodegenerative conditions, such as Parkinson's disease, where non-motor symptoms are common and impactful, disease-specific and symptom-specific scales such as Parkinson's Disease Fatigue Scale [47], King's Parkinson's Disease Pain Scale [48] and Parkinson's Disease Sleep Scale [49] have clinical utility [50]. As a result, in this review we also considered the intended purpose of each of the tools utilised to evaluate non-motor symptoms. In using assessment tools that are specific to, or adapted for, the population we are evaluating, we are better able to determine the prevalence and progression of non-motor symptoms, whilst accounting for the progressive disability and speech impairments of ALS that may influence responses. Including revised impairment thresholds in well-established generic measures can also be a viable alternative to mitigate potential confounding effects of ALS.

However, measures designed to evaluate the non-motor features of ALS and other neurodegenerative diseases have not been frequently employed in clinical care, research or trial design [10]. Potential barriers to their use include a limited evidence base compared to established measures and additional time burden for participants. These obstacles can be addressed with further research into the validity, reliability and utility of brief measures adapted to assess these non-motor symptoms within the context of neurodegenerative diseases. 


\section{Strengths and weaknesses of the study, and future recommendations}

This study indicates that non-motor symptoms have not been comprehensively or consistently evaluated within clinical trials of ALS. An improved understanding of the frequency that these symptoms occur, and their contribution to acquired disability, will enable us to provide a more holistic overview of an ALS diagnosis and potential impact of investigative medicinal products. The key strength of this study is that it provides a comprehensive evaluation of ALS trials completed, published or registered since 1994. In addition, we provide a detailed overview and critique of the assessment tools used to explore these non-motor symptoms in the included trials. However, a weakness of the current work is the focus on a limited number of non-motor symptoms in ALS, namely neuropsychiatric, cognitive and behavioural changes, pain, disordered sleep and fatigue and problematic saliva. However, the scope of non-motor symptoms can be extended in future studies to assessment of other symptoms including gastrointestinal issues, dysphagia and sexual dysfunction.

We recommend that future clinical trials should include non-motor outcome measures. In addition, more research should focus on the association between these symptomatic outcomes and the potential benefit experienced by trial participants. Whilst it is appropriate that measurement of change in functional decline and improvement in survival remain as primary outcomes in confirmatory trials, we recommend future trials include disease-specific secondary outcome measures to establish the effect of investigative medicinal products on non-motor symptoms to enable a more complete profile of how a candidate drug may affect pwALS. Cognitive assessments are already gradually receiving greater prominence in trial design [11], reflective of Airlie House guidance encouraging the use of cognitive or behavioural functioning as primary or secondary outcome measures [9].

Evaluation of the key non-motor symptoms considered in this study was primarily using tools which may not be suitable for people with ALS, or generic assessments of physical function or quality of life where symptom-specific changes were not apparent. These non-motor symptoms should be evaluated with assessment tools which are ALS-specific or validated for use in people with ALS, including disease-specific impairment thresholds where possible.

Future work should focus on evaluating the prevalence and impact of each of these non-motor symptoms in people with ALS. This research should also explore the comparison of different assessment tools for each non-motor symptom. Providing recommendations for assessment tools that are suitable to evaluate non-motor symptoms, or the availability of disease-specific impairment thresholds, will be a useful and relevant direction for future work.

Supplementary Information The online version contains supplementary material available at https://doi.org/10.1007/s00415-021-10651-1.

Author contributions $\mathrm{SP}$ and $\mathrm{EB}$ conceived the project. $\mathrm{EB}, \mathrm{ZH}$ and DF completed data analysis. SP, EB, DF and ZH drafted the manuscript. All the authors reviewed the manuscript.

Funding The Euan MacDonald Centre for Motor Neurone Disease Research, S.C. is supported by the UK Dementia Research Institute (DRI), which receives its funding from UK DRI Ltd, funded by the MRC, Alzheimer's Society and Alzheimer's Research UK.

Availability of data and material Supplementary Material Document 1 .

\section{Declarations}

Conflicts of interest Professor Carson is a paid editor at Journal of Neurology, Neurosurgery and Psychiatry, and gives independent testimony in Court on a range of neuropsychiatric topics.

Ethical standard statement The authors confirm that this article complies with ethical standards.

Open Access This article is licensed under a Creative Commons Attribution 4.0 International License, which permits use, sharing, adaptation, distribution and reproduction in any medium or format, as long as you give appropriate credit to the original author(s) and the source, provide a link to the Creative Commons licence, and indicate if changes were made. The images or other third party material in this article are included in the article's Creative Commons licence, unless indicated otherwise in a credit line to the material. If material is not included in the article's Creative Commons licence and your intended use is not permitted by statutory regulation or exceeds the permitted use, you will need to obtain permission directly from the copyright holder. To view a copy of this licence, visit http://creativecommons.org/licenses/by/4.0/.

\section{References}

1. Fang T, Jozsa F, Al-Chalabi A (2017) Nonmotor symptoms in amyotrophic lateral sclerosis: a systematic review. Int Rev Neurobiol 134:1409-1441

2. Günther $\mathrm{R}$ et al (2016) Non-motor symptoms in patients suffering from motor neuron diseases. Front Neurol 7:117

3. Prell T et al (2019) Disease progression impacts health-related quality of life in amyotrophic lateral sclerosis. J Neurol Sci 397:92-95

4. Kanner AM, Barry JJ (2003) The impact of mood disorders in neurological diseases: should neurologists be concerned? Epilepsy Behav 4:3-13

5. Lou J-S et al (2003) Fatigue and depression are associated with poor quality of life in ALS. Neurology 60(1):122-123

6. Raheja D et al (2016) Patient-reported problematic symptoms in an ALS treatment trial. Amyotrophic Lateral Scler Frontotemp Degener 17(3-4):198-205

7. Christidi $\mathrm{F}$ et al (2018) Clinical and radiological markers of extra-motor deficits in amyotrophic lateral sclerosis. Front Neurol 9:1005 
8. National Institute for Health and Care Excellence (2016) Motor neurone disease: assessment and management. [NICE Guideline No. 42]. https://www.nice.org.uk/guidance/ng42

9. Van Den Berg LH et al (2019) Revised Airlie House consensus guidelines for design and implementation of ALS clinical trials. Neurology 92(14):e1610-e1623

10. Ng L et al (2017) Symptomatic treatments for amyotrophic lateral sclerosis/motor neuron disease. Cochrane Database Syst Rev $1: 1-28$

11. Beswick E et al (2020) A systematic review of neuropsychiatric and cognitive assessments used in clinical trials for amyotrophic lateral sclerosis. J Neurol 267:1-12

12. Miller RG, Mitchell JD, Moore DH (2012) Riluzole for amyotrophic lateral sclerosis (ALS)/motor neuron disease (MND). Cochrane Database Syst Rev 3:1-28

13. van Eijk RPA, Kliest T, van den Berg LH (2020) Current trends in the clinical trial landscape for amyotrophic lateral sclerosis. Curr Opin Neurol 33(5):655-661. https://doi.org/10.1097/WCO. 0000000000000861

14. Hammer EM et al (2008) Validity of the ALS-DepressionInventory (ADI-12) - a new screening instrument for depressive disorders in patients with amyotrophic lateral sclerosis. J Affect Disord 109(1-2):213-219

15. Niven E et al (2015) Validation of the Edinburgh Cognitive and Behavioural Amyotrophic Lateral Sclerosis Screen (ECAS): a cognitive tool for motor disorders. Amyotrophic Lateral Scler Frontotemp Degener 16(3-4):172-179

16. Gibbons CJ et al (2011) Rasch analysis of the hospital anxiety and depression scale (HADS) for use in motor neurone disease. Health Qual Life Outcomes 9:82

17. Cedarbaum JM et al (1999) The ALSFRS-R: a revised ALS functional rating scale that incorporates assessments of respiratory function. BDNF ALS Study Group (Phase III). J Neurol Sci $169(1-2): 13-21$

18. Smith RA et al (2018) Assessment of bulbar function in amyotrophic lateral sclerosis: validation of a self-report scale (Center for Neurologic Study Bulbar Function Scale). Eur J Neurol 25(7):907-e66

19. Chang VT, Hwang SS, Feuerman M (2000) Validation of the Edmonton symptom assessment scale. Cancer 88(9):2164-2171

20. Williams JB (1988) A structured interview guide for the Hamilton Depression Rating Scale. Arch Gen Psychiatry 45(8):742-747

21. Kaufer DI et al (2000) Validation of the NPI-Q, a brief clinical form of the Neuropsychiatric Inventory. J Neuropsychiatry Clin Neurosci 12(2):233-239

22. Posner $\mathrm{K}$ et al (2008) Columbia-suicide severity rating scale (C-SSRS). Columbia University Medical Center, New York, $\mathrm{p}$ 10

23. Zigmond AS, Snaith RP (1983) The hospital anxiety and depression scale. Acta Psychiatr Scand 67(6):361-370

24. Simmons $Z$ et al (2006) The ALSSQOL: balancing physical and nonphysical factors in assessing quality of life in ALS. Neurology 67(9):1659-1664

25. Jenkinson RF, Brennan C, Crispin MS (2000) Evidence for the validity and reliability of the ALS assessment questionnaire: the ALSAQ-40. Amyotroph Lateral Scler Other Motor Neuron Disord 1(1):33-40

26. Ware JE Jr, Sherbourne CD (1992) The MOS 36-item short-form health survey (SF-36): I. Conceptual framework and item selection. Med Care 6:473-483

27. Rabin R, Charro FD (2001) EQ-SD: a measure of health status from the EuroQol Group. Ann Med 33(5):337-343

28. Cohen SR et al (1995) The McGill Quality of Life Questionnaire: a measure of quality of life appropriate for people with advanced disease. A preliminary study of validity and acceptability. Palliat Med 9(3):207-219

29. Bergner M et al (1981) The sickness impact profile: development and final revision of a health status measure. Medical Care 8:787-805

30. Woolley SC et al (2010) Detecting frontotemporal dysfunction in ALS: utility of the ALS Cognitive Behavioral Screen (ALSCBSTM). Amyotroph Lateral Scler 11(3):303-311

31. Nasreddine ZS et al (2005) The Montreal Cognitive Assessment, MoCA: a brief screening tool for mild cognitive impairment. J Am Geriatr Soc 53(4):695-699

32. Mioshi E et al (2006) The Addenbrooke's Cognitive Examination Revised (ACE-R): a brief cognitive test battery for dementia screening. Int J Geriatr Psychiatry 21(11):1078-1085

33. Woolley SC, Moore DH, Katz JS (2010) Insight in ALS: awareness of behavioral change in patients with and without FTD. Amyotroph Lateral Scler 11(1-2):52-6

34. Werner P et al (2014) Help-seeking for dementia: a systematic review of the literature. Alzheimer Dis Assoc Disord 28(4):299-310

35. Kertesz A, Davidson W, Fox H (1997) Frontal behavioral inventory: diagnostic criteria for frontal lobe dementi. Can J Neurol Sci 24(1):29-36

36. Couratier P, Torny F, Lacoste M (2006) Functional rating scales for amyotrophic lateral sclerosis. Rev Neurol (Paris) 162(4):502-7

37. Weiss MD et al (2016) A randomized trial of mexiletine in ALS: Safety and effects on muscle cramps and progression. Neurology 86(16):1474-81

38. Johns MW (1991) A new method for measuring daytime sleepiness: the Epworth sleepiness scale. Sleep 14(6):540-545

39. Buysse DJ et al (1989) The Pittsburgh Sleep Quality Index: a new instrument for psychiatric practice and research. Psychiatry Res 28(2):193-213

40. Schwartz JE, Jandorf L, Krupp LB (1993) The measurement of fatigue: a new instrument. J Psychosom Res 37(7):753-762

41. Simmons Z, Felgoise SH, Rodriguez JL, Walsh SM, Bremer BA, Stephens HE (2010) Validation of a shorter ALS-specific quality of life instrument: the ALSSQOL-R. Neurology 74(suppl 2):A177-A178

42. Felgoise $\mathrm{SH}$ et al (2018) Amyotrophic lateral sclerosis-specific quality of life-short form (ALSSQOL-SF): a brief, reliable, and valid version of the ALSSQOL-R. Muscle Nerve 58(5):646-654

43. Jenkinson C et al (1999) Development and validation of a short measure of health status for individuals with amyotrophic lateral sclerosis/motor neurone disease: the ALSAQ-40. J Neurol 246(3):II16-II21

44. Crockford $\mathrm{C}$ et al (2018) Measuring reliable change in cognition using the Edinburgh Cognitive and Behavioural ALS Screen (ECAS). Amyotrophic Lateral Scler Frontotemp Degener 19(1-2):65-73

45. Strong MJ et al (2009) Consensus criteria for the diagnosis of frontotemporal cognitive and behavioural syndromes in amyotrophic lateral sclerosis. Amyotroph Lateral Scler 10(3):131-146

46. Hobson EV, McDermott CJ (2016) Supportive and symptomatic management of amyotrophic lateral sclerosis. Nat Rev Neurol 12(9):526

47. Brown R et al (2005) The Parkinson fatigue scale. Parkinsonism Relat Disord 11(1):49-55

48. Chaudhuri KR et al (2015) King's Parkinson's disease pain scale, the first scale for pain in PD: an international validation. Mov Disord 30(12):1623-1631

49. Trenkwalder C et al (2011) Parkinson's disease sleep scale-validation of the revised version PDSS-2. Mov Disord 26(4):644-652

50. Schapira AH, Chaudhuri KR, Jenner P (2017) Non-motor features of Parkinson disease. Nat Rev Neurosci 18(7):435 
51. Kurt A et al (2007) Depression and anxiety in individuals with amyotrophic lateral sclerosis. CNS Drugs 21(4):279-291

52. Díaz JL et al (2016) Effect of a short-term psychological intervention on the anxiety and depression of amyotrophic lateral sclerosis patients. J Health Psychol 21(7):1426-1435

53. Silva-Moraes MH et al (2020) Suicidal behavior in individuals with amyotrophic lateral sclerosis: a systematic review. J Affect Disord 277:688-696

54. McHutchison CA et al (2020) Relationship between neuropsychiatric disorders and cognitive and behavioural change in MND. J Neurol Neurosurg Psychiatry 91(3):245-253

55. Benbrika $S$ et al (2019) Cognitive, emotional and psychological manifestations in amyotrophic lateral sclerosis at baseline and overtime: a review. Front Neurosci 13:951

56. Caga J et al (2019) The impact of cognitive and behavioral symptoms on ALS patients and their caregivers. Front Neurol 10:192

57. Goldstein LH, Abrahams S (2013) Changes in cognition and behaviour in amyotrophic lateral sclerosis: nature of impairment and implications for assessment. Lancet Neurol 12(4):368-380

58. Radakovic R et al (2016) Multidimensional apathy in ALS: validation of the Dimensional Apathy Scale. J Neurol Neurosurg Psychiatry 87(6):663-669

59. Chiò A et al (2012) Pain in amyotrophic lateral sclerosis: a population-based controlled study. Eur J Neurol 19(4):551-555

60. Aksu S, Citak-Karakaya I (2002) Effect of exercise therapy on pain complaints in patients with amyotrophic lateral sclerosis. Pain Clin 14(4):353-359

61. McClelland S III et al (2008) Intrathecal baclofen for spasticityrelated pain in amyotrophic lateral sclerosis: efficacy and factors associated with pain relief. Muscle Nerve 37(3):396-398

62. Boentert M (2019) Sleep disturbances in patients with amyotrophic lateral sclerosis: current perspectives. Nat Sci Sleep 11:97

63. Katzberg HD et al (2013) Effects of noninvasive ventilation on sleep outcomes in amyotrophic lateral sclerosis. J Clin Sleep Med 9(4):345-351

64. Chio A, Cicolin A (2021) Sleep in ALS: more than discomfort or respiratory breathing disorder. BMJ Publishing Group Ltd.

65. Lo Coco D et al (2012) Sleep-wake problems in patients with amyotrophic lateral sclerosis: implications for patient management. Neurodegener Dis Manag 2(3):315-324

66. McElhiney M et al (2009) Prevalence of fatigue and depression in ALS patients and change over time. J Neurol Neurosurg Psychiatry 80(10): 1146-1149

67. Gibbons C, Pagnini F, Friede T, Young CA (2018) Treatment of fatigue in amyotrophic lateral sclerosis/motor neuron disease. Cochrane Database Syst Rev 1(1):CD011005. https://doi.org/10. 1002/14651858.CD011005.pub2
68. Bradley WG et al (2001) Current management of ALS: comparison of the ALS CARE database and the AAN practice parameter. Neurology 57(3):500-504

69. Pearson I, Glasmacher SA, Newton J, Beswick E, Mehta AR, Davenport R, Chandran S, Pal S (2020) CARE-MND consortium. The prevalence and management of saliva problems in motor neuron disease: a 4-year analysis of the Scottish motor neuron disease register. Neurodegener Dis 20(4):147-152. https://doi.org/10. $1159 / 000514615$

70. Palmieri A et al (2010) Quality of life and motor impairment in ALS: Italian validation of ALSAQ. Neurol Res 32(1):32-40

71. Oh J et al (2017) Translation and psychometric evaluation of a Korean version of the amyotrophic lateral sclerosis-specific quality of life-revised. Amyotrophic Lateral Scler Frontotemp Degener 18(1-2):92-98

72. Carvajal A et al (2013) The Spanish version of the Edmonton Symptom Assessment System-revised (ESAS-r): first psychometric analysis involving patients with advanced cancer. J Pain Symptom Manag 45(1):129-136

73. Mekala $\mathrm{S}$ et al (2020) Dementia diagnosis in seven languages: the Addenbrooke's Cognitive Examination-III in India. Arch Clin Neuropsychol 35(5):528-538

74. Tremolizzo L, Lizio A, Santangelo G, Diamanti S, Lunetta C, Gerardi F, Messina S, La Foresta S, Riva N, Falzone Y, Filippi M, Woolley SC, Sansone VA, Siciliano M, Ferrarese C, Appollonio I (2020) ALS-CBS Italian study group. ALS cognitive behavioral screen (ALS-CBS): normative values for the Italian population and clinical usability. Neurol Sci 41(4):835-841. https://doi.org/ 10.1007/s10072-019-04154-1

75. Mora JS et al (2018) Spanish adaptation of the edinburgh cognitive and behavioral amyotrophic lateral sclerosis screen (ECAS). Amyotrophic Lateral Scler Frontotemp Degener 19(1-2):74-79

76. Poletti B et al (2016) The validation of the Italian Edinburgh cognitive and behavioural ALS screen (ECAS). Amyotrophic Lateral Scler Frontotemp Degener 17(7-8):489-498

77. Alberici A et al (2007) The Frontal Behavioural Inventory (Italian version) differentiates frontotemporal lobar degeneration variants from Alzheimer's disease. Neurol Sci 28(2):80-86

78. Oda E et al (1996) Reliability and factorial structure of a rating scale for amyotrophic lateral sclerosis. No to shinkei. Brain Nerve 48(11):999-1007

79. Hita-Contreras F et al (2014) Reliability and validity of the Spanish version of the Pittsburgh Sleep Quality Index (PSQI) in patients with fibromyalgia. Rheumatol Int 34(7):929-936 